\title{
LES HÉBRAÏSMES ET LES SLAVISMES DU YIDDISH ET LA CRISTALLISATION D'UNE IDENTITÉ JUIVE EST-EUROPÉENNE
}

\author{
Cyril Aslanov ${ }^{1}$
}

\begin{abstract}
At the beginning of the twentieth century, the defenders of Yiddish tried to standardize the language according to a "Lithuanian" norm. This practical endeavor had its scientific legitimization through the monumental work of the prominent linguist Max Weinreich who demonstrated that the coexistence of the various sub-systems - Germanic, Hebrew, Slavic - within Yiddish obeyed rigorous rules and was far from being macaronic, as detractors of Yiddish used to insinuate. However, Weinreich's schematic approach fails to explain the correlation between the huge proportion of Hebraisms in Yiddish and the strong impact of Slavic adstrates. From a diachronic viewpoint, the Hebraization and the Slavization of Yiddish may correspond to a period of instability in the linguistic system, which took place between the end of the Middle Age and the early modern times. This study deals with the combined impact of Hebraization and Slavization within the system of Yiddish as well as from the external perspective of sociolinguistics.
\end{abstract}

Key words: Diachronic linguistics; sociolinguistics; languages in contact; linguistic interference; Yiddish; Eastern Europe.

Resumo: No início do século XX, os defensores do ídiche procuraram estandardizar a língua segundo a norma "lituana". Este projeto prático recebeu a sua legitimação científica na obra monumental do eminente lingüista Max Weinreich que demonstrou que a coexistência dos vários sub-sistemas - o germânico, o hebraico, o eslavo - dentro do ídiche obedecia a regras rigorosas e não tinha nada de macarrónico, como 0 insinuavam os detractores desta língua. Contudo, a abordagem esquemática de Weinreich não logrou explicar a correlação entre a considerável proporção de hebraísmos no ídiche e 0 forte impacto dos adstratos eslávos. Desde o ponto de vista diacrônico, a hebraização e a eslavização do ídiche podem corresponder a um período de inestabilidade no sistema lingüístico que aconteceu entre o final da Idade Média e o princípio dos tempos modernos. Este estudo trata do impacto conjunto da hebraização e da eslavização tanto desde ponto de vista interno do sistema da língua ídiche, quanto desde a perspectiva externa da sociolingüística.

Palavras-chave: lingüística diacrônica; sociolingüística; línguas em contato; interferência lingüística; ídiche; Europa oriental.

\section{INTRODUGTION}

\footnotetext{
1 Universidade Hebraica de Jerusalém.
} 
La problématique du métissage des langues se rattache à la créolistique dont le fondateur Hugo Schuchardt fut aussi le premier à donner droit de cité au concept de langue mixte (Spitzer, 1928: 150-163). ${ }^{2}$ Les méthodes de la créolistique ou du moins des méthodes qui y sont apparentées ont déjà été adaptées à l'étude du yiddish, notamment en ce qui concerne la relexification et le changement motivé par le contact linguistique (Eggers, 1998; Prince, 2001). Dans cette perspective, le métissage du yiddish consisterait dans le fait que cette langue a conservé des structures grammaticales germaniques tout en modifiant considérablement son lexique à l'aide d'emprunts massifs à l'hébreu et aux langues slaves. Selon le même principe, mais en sens inverse, Paul Wexler a voulu voir dans le yiddish une langue slave revêtue d'un vernis germanique (Wexler, 1991; 2002). Reprenant en partie la vieille thèse de l'origine khazare des Ashkénazes, il étendit son scepticisme à l'histoire externe de la langue dans un ouvrage contesté intitulé The Ashkenazic Jews: A Slavic-Turkic People in Search of a Jewish Identity (Wexler, 1993). Quoi qu'il en soit, ces débats sur la slavité du yiddish - slavité des structures profondes, comme le voudrait Wexler, ou slavité d'emprunt ou de contact, comme le reconnaissent la plupart des yiddishisants (Weinreich, 1980: 525-598) - risquent de faire oublier que la langue yiddish est assurément beaucoup plus riche d'hébraïsmes que de slavismes et qu'elle dépasse de loin toutes les autres judéo-langues par la quantité d'emprunts lexicaux qu'elle a contractés auprès de la langue sainte.

Pour rendre compte du double métissage linguistique qui affecta le yiddish et l'identité linguistique ashkénaze au moment du transfert d'Ashkenaz I (les pays germanophones) à Ashkenaz II (les pays slaves) (Weinreich, 1980: 3-4), il importe de ne pas séparer les phénomènes de la slavisation de la dynamique de l'hébraïsation. La tendance à considérer isolément la slavisation et l'hébraïsation caractérise notamment l'approche mise en œuvre par Max Weinreich dans son histoire de la langue yiddish à laquelle nous venons de faire référence. Bien que cette œuvre colossale soit une référence difficilement dépassable, la façon dont Weinreich rend compte de la coexistence des sous-systèmes à l'intérieur de la langue présente l'inconvénient d'isoler outre mesure chacune des composantes du cocktail linguistique yiddish, de sorte que le lecteur a parfois du mal à percevoir la dynamique de la coexistence des sous-systèmes au sein du système général de la langue. De plus, la volonté de promouvoir une norme poussa Weinreich à modéliser de façon un peu trop formaliste les faits qu'il décrivait, comme si le

\footnotetext{
2 Schuchardt s'intéressa spécifiquement aux hybridations linguistiques entre l'allemand et les langues slaves (le tchèque notamment) dans une brève étude d'une densité exceptionnelle (Schuchardt, 1884). Mais il ne semble pas avoir pris connaissance du potentiel que représentait le yiddish oriental pour ses recherches sur les langues mixtes germano-slaves.
} 
foisonnement anarchique des différentes variétés du yiddish avait effrayé ce partisan de l'uniformisation et de la standardisation de la langue.

Dans une perspective diatopique et diachronique, la comparaison du yiddish oriental avec le judéo-allemand occidental (celui qui ne participa à la translation d'Ashkenaz I à Ashkenaz II) confirme nettement que celui-là se distingue de celui-ci par les facteurs conjugués de la slavisation et de l'hébraïsation. Certes le yiddish occidental possédait naguère une variété très riche en hébraismes, mais il s'agissait d'un sociolecte cryptolalique restreint à la catégorie professionnelle des marchands de bestiaux (loshen $\infty$ koudesh) d'Alsace, de Bade et de Suisse (Guggenheim-Grinberg, 1954). En revanche, la langue commune des communautés rurales d'Ashkenaz I se caractérisait par la relative maigreur de la composante hébraïque et naturellement par l'absence presque totale de slavismes. ${ }^{3}$

Nous voudrions reconsidérer la question des hébraïsmes et des slavismes du yiddish oriental en essayant de démontrer que les deux phénomènes sont éminemment liés. Mais il importe de définir la nature exacte de ce lien. S'agitil d'un phénomène touchant au système interne de la langue ou d'une dynamique concernant la sociolinguistique historique? Dans la première hypothèse, l'hébraïsation intense serait survenue lors d'une phase d'instabilité de l'histoire du yiddish, à l'époque où une koinè judéo-allemande (protoyiddish oriental) fraîchement cristallisée ou en voie de cristallisation se trouva confrontée à l'environnement alloglotte d'Ashkenaz II. Dans la seconde hypothèse, l'hébraïsation serait une stratégie délibérée consécutive au choc culturel résultant de la rencontre entre les Ashkénazes germanophones venus d'Allemagne et les Juifs slavophones, descendants des populations juives byzantines installées en Russie kiévienne depuis le $\mathrm{X}^{\mathrm{e}}$ siècle au moins.

\section{L'HÉBRAÏSATION, GOROLLAIRE DE LA SLAVISATION ?}

Lorsque les premiers immigrants juifs allemands s'installèrent en Pologne et en Lithuanie vers la fin du XIII ${ }^{\mathrm{e}}$ siècle, il est fort probable que la koinè judéoallemande qui constitue la base germanique du yiddish oriental ne s'était pas encore cristallisée. ${ }^{4}$ Comme l'enseigne la sociolinguistique, la constitution d'une

3 Les deux seuls slavismes du yiddish occidental sont kauletsch, nom d'une sorte de brioche au fromage et nebex/ nevex"infortuné". Alors que le premier ne se rencontre qu'en yiddish occidental, le second est largement attesté à l'Est (Weinreich, 1980: 542-543).

4 Cette affirmation heurte de front la thèse weinreichienne selon laquelle le yiddish se serait constitué durant le Haut Moyen Âge au terme d'une synthèse entre divers composantes linguistiques: le roman des Juifs venus d'Italie ou de France, l'hébreu et le vieux haut allemand (Weinreich, 1980: 424). Mais Weinreich était mû par un a priori idéologique qui consistait à supposer que chaque communauté juive aurait développé une variante spécifiquement juive de la langue coterritoriale parlée par les non-juifs. 
koinè supradialectale dans les milieux d'immigrants est un phénomène postérieur à l'immigration (Siegel, 1985). Même si le premier niveau de koinéisation $\mathrm{du}$ yiddish concerne essentiellement les dialectes allemands mis en présence dans le nouveau contexte d'Ashkenaz II, la toile de fond de cette première synthèse était assurément un environnement linguistique alloglotte, qu'il s'agisse du contexte non-juif ou des Juifs slavophones d'Ukraine et de Pologne dont la présence est garantie depuis le Haut Moyen Age.

Un exemple de l'impact du slave dans le processus de koinéisation du yiddish oriental nous est fourni par l'aboutissement des voyelles [ø] bref et long, [y] bref et long et les diphtongues [iu] et [öu] du moyen haut allemand. En yiddish, ces phonèmes deviennent respectivement [e] (töhter $>$ tekhter ${ }^{5}$ "filles", [ei] (boese > beyz "en colère"); [i ] (gelücke > glik "chance"); [i] (müede > mid "fatigué"); [ai] (liute > layt "gens"); [ei] (vröude > freyd "joie"). ${ }^{6}$ Selon Weinreich, la tendance à la neutralisation des palatales arrondies remonterait à la préhistoire du yiddish, c'est-à-dire à l'époque où le protoyiddish était coterritorial aux dialectes du moyen haut allemand (Weinreich, 1980: 464). Un peu plus loin, ce savant se fonde sur un argumentum ex silentio lorsqu'il fonde son affirmation de l'absence de palatales arrondies en yiddish médiéval sur de vieilles graphies judéo-allemandes du XIV siècle (Weinreich, 1980 : 465). Or le parallèle d'autres judéo-langues révèle que les graphies en caractères hébraïques ne notent pas forcément la valeur palatale de [y] ou de [ø]. Le témoignage de la graphie ne saurait donc constituer une preuve suffisante en faveur de la non-existence de $[y]$ et de $[\varnothing]$ dans le moyen haut allemand des Juifs d'Allemagne au Moyen Age.

Nous voudrions nous démarquer de l'approche monolithique de Weinreich en supposant que le protoyiddish parlé par les Juifs d'Allemagne médiévale variait considérablement selon les régions où il était pratiqué (comme les dialectes du moyen haut allemand). Dans cette hypothèse, c'est seulement au cours de la koinéisation survenue en terres slaves que les voyelles palatales

Comme le recommande Robert $\mathrm{D}$. King dans un article sur la morphologie du protoyiddish, la reconstitution du protoyiddish n'a de sens que si elle est précédée d'une reconstitution du protoyiddish oriental d'une part et du protoyiddish occidental d'autre part (King, 1987: 75).

5 Nous avons choisi de translittérer les mots du yiddish au moyen du système d'équivalences officiellement adopté par le YIVO Institute for Jewish Research. Voir le site yivoinstitute.org/ yiddish/ alefbeys.htm.

6 Seuls quelques dialectes secondaires du yiddish oriental font apparaitre des voyelles palatales arrondies. Le maintien de [y] dans le parler yiddish de Brajnsk est imputable à la position-charnière de cet isolat entre les dialectes nord-orientaux (dialectes ayant préservé $[u]<m . h . a$. [u] et les dialectes nord-centraux du yiddish (dialectes ayant palatalisé ce [u] en [i] (Herzog, 1965 : 167). Sur le maintien de [øi] en yiddish de Courlande (dialecte nord-oriental), voir Herzog (1965: 170) et Jacobs (1990 : 64-65). 
arrondies auraient disparu. ${ }^{7}$ Cela est d'autant plus vraisemblable que les processus de koinéisation supposent une sélection des normes qui va dans le sens de la simplification du système. Dans ce cas précis, la perte de [ø], [y] et [öu] peut être imputée à l'action de l'adstrat slave. Rappelons en effet que mis à part quelques exceptions dans certains dialectes parlés naguère dans la zone de transition entre l'ukrainien et le biélorusse (Kuraskiewicz, 1963 : 68-69), les langues slaves coterritoriales au yiddish oriental ne possèdent pas de voyelles palatales arrondies dans leur système phonologique.

La simplification du système phonologique consécutive à la koinéisation a exercé un impact décisif sur le système morphologique de la langue. Un grand nombre de formes et de lexèmes tombèrent en désuétude du fait même de leur incompatibilité avec les schèmes morphophonétiques du système rénové. Les lacunes ainsi créées dans le système de la langue ont été comblées par un recours massif à deux sources de renouvellement lexical: le superstrat hébreu et l'adstrat slave (substrat si l'on considère qu'un certain nombre de Juifs slavophones finirent par adopter la langue prestigieuse des immigrants venus d'Ashkenaz I). Ainsi la catégorie morphologique des verbes suffixés en -enen constitue un exemple éloquent de relexification motivée en partie par la perte de lexèmes allemands sans doute ressentis comme peu commodes par les usagers de la langue. Mis à part le cas de rekhenen "compter" formé sur une racine germanique, de leyenen "lire" et de plankhenen "pleurer", tous deux d'origine romane, et de davenen "prier" dont l'étymologie est très disputée, il apparaît que -enen a servi à créer des verbes yiddish sur la base d'une racine hébraïque: badk-enen "vérifier" à partir du verbe badaq ; ganv-enen "voler" à partir du verbe ganav ou du nom d'agent gannav "voleur"; harg-enen "tuer" à partir du verbe harag; xasm-enen "signer" à partir de katam (prononcé khosam dans la tradition ashkénaze); pask-enen "décider" à partir de pasaq.

Ces verbes sont hybrides puisqu'ils sont composés d'une racine hébraïque et d'un suffixe complexe où l'on est tenté de reconnaitre l'infixe $\{-n-\}$, si productif dans la formation des verbes slaves. ${ }^{8}$ Ces néologismes du yiddish ori

\footnotetext{
${ }^{7}$ Dans sa description de la genèse du vocalisme des dialectes yiddish de la Pologne septentrionale, Herzog établit une correspondance entre le vocalisme du yiddish nord-oriental ("lithuanien") et celui du proto-yiddish nord-oriental (Herzog, 1965 : 164).

8 L'identification de l'élément $\{-n-\}$ avec un composant morphologique slave corroborée par la valeur semelfactive ou inchoative qu'il revêt dans le système du verbe des langues slaves. De fait, les verbes en \{-enen\} formés sur des racines hébraïques ont tous une valeur semelfactive. L'analyse qui consiste à reconnaître un infixe slave $\{-n\}$ dans le suffixe $\{$-enen $\}$ est corroborée par le parallèle du suffixe $\{$-even $\}$ où l'élément\{-ev\} est sans aucun doute un emprunt au slave (Weinreich, 1980: 531; 621). Quant aux verbes rexenen "compter", leyenen "lire", plankhenen (planyenen en yiddish occi-dental) "pleurer" et davenen "prier", ils ne peuvent être considérés comme faisant partie de la catégorie des verbes en $\{$-enen\} que dans une perspective strictement synchronique. Du point de vue diachronique, leur formation s'explique très
} 
ental ont fourni des alternatives simples et maniables à des verbes germaniques peu commodes à l'usage:" badkenen "vérifier" a remplacé brüefen/ prüefen qui comportait un $[\mathrm{y}]$ long difficilement prononçable après la perte de [y] consécutive au processus de koinéisation en terres slaves et après la transphonologisation qui aboutit à la perte du critère de longueur dans le système phonologique du yiddish oriental; hargenen "tuer" s'est substitué à tæeten dont le $[\varnothing]$ fait également partie des phonèmes disparus au cours de la cristallisation du yiddish oriental. De son côté, l'emploi du verbe khasmenen "signer" au lieu du verbe moyen-haut-allemand underschrîben est peut-être due au fait que le yiddish oriental a profondément bouleversé le système des verbes composés hérité de l'allemand, le plus souvent sous l'influence de l'adstrat slave (Wexler, 1964; Weinreich, 1980: 529-530). De plus, en moyen haut allemand, underschrîben n'a pas tout a fait le sens de l'allemand moderne unterschreiben "signer". Ce verbe signifie plutôt "consigner par écrit, affirmer par l'écrit" au sens que niederschreiben revêt aujourd'hui en allemand.

Pour rendre compte du remplacement de underschrîben par khasmenen, on peut aussi avancer une explication d'ordre pragmatique. Dans le monde slave tout comme dans le monde juif traditionnel, les accords commerciaux se faisaient par oral, sur la fois de la parole donnée. Par conséquent, l'une des seules aires d'activité où la signature était de mise était celle de la rédaction des contrats de mariage ou des lettres de répudiation, documents qui ressortissent bien évidemment à la sphère cultuelle d'expression hébraïque (araméenne dans le cas des contrats de mariage). Le même genre d'explication vaut également pour paskenen "décider". Rappelons en effet qu'à l'origine, le verbe hébreu pasaq servait à désigner l'activité décisionnaire des rabbins dans le contexte de la jurisprudence juive traditionnelle.

différemment : voyelle d'anaptyxe dans le cas de rexenen (cf. allemand rechnen) et peut-être aussi dans le cas de davenen dont une variante davnen est attestée dans certains dialectes du yiddish; réactivation redondante de la marque morphologique dans le cas de leyenen $\leftarrow$ leyen $<$ roman legere et de plankhenen/ planyenen $\leftarrow{ }^{*}$ plankhenl planyen $<$ roman plangere. Quant à dükhene $(n)$ "prononcer la bénédiction sacerdotale", il n'est attesté qu'en yiddish occidental et il doit bien entendu être analysé comme une combinaison de dükhen < hébreu dukhan "tribune de la synagogue d'où les cohanim prononcent la bénédiction sacerdotale" et de la désinence d'infinitif \{-en\}. La formation d'un verbe yiddish moyennant la suffixation de $\{$-en\} à la racine hébraïque est également attestée en yiddish orientale. Cf. par exemple mekn "effacer" < ma 'aq; shekhtn "abattre un animal" < sha'aq. Ces deux verbes doivent appartenir à une très ancienne strate de la langue, comme le montre l'application des règles du Umlaut, normalement réservée à la composante germanique du yiddish (Weinreich, 1980: 625).

${ }^{9}$ Le remplacement d'une forme nominale ou verbale compliquée par une alternative plus simple est un des mécanismes de base du renouvellement des langues. La spécificité des cas dont nous traitons ici tient au fait que dans les formes germaniques remplacées par des hébraïsmes, ce n'est pas la partie désinentielle du verbe qui posait un problème, mais plutôt le phonétisme de la partie radicale. 
Quant à ganvenen "voler", il est très probable que sa formation a été motivée par une stratégie cryptolalique. Toutefois la valeur semelfactive attachée au suffixe \{-enen\} a pu jouer un rôle dans le choix de ce verbe aux dépens des aboutissements du verbe moyen-haut-allemand stëln / stëlen "voler".

Des facteurs internes au système de la langue se sont donc conjugués avec des motivations sémantiques et pragmatiques ponctuelles pour favoriser le remplacement de verbes germaniques par des verbes combinant une racine hébraïque et un infixe verbal slave. Mais l'impact de l'hébreu et du slave ne concerne pas seulement la dimension lexicale. Il touche aussi à des pans entiers des œuvres vives de la langue. Ainsi, il est très vraisemblable que la perte du préterit que Weinreich attribuait à des dynamiques sociolinguistiques inhérentes à Ashkenaz I (Weinreich, 1980: 517-518) soit en fait consécutive à la koinéisation en contexte slave. On sait en effet que le polonais, tout comme beaucoup d'autres langues slaves et à la différence de l'allemand, ne possède plus ni prétérit ni parfait, mais une seule forme de passé d'origine périphrastique. ${ }^{10}$ En réduisant ses temps du passé au seul parfait, le système verbal du yiddish oriental convergea non seulement avec les langues slaves coterritoriales, mais aussi avec l'hébreu qui lui aussi ne possède qu'un temps passé. ${ }^{11}$

Un autre aspect de l'action conjuguée du superstrat hébreu et de l'adstrat slave concerne les cas de concurrence entre les alternatives hébraïques et slaves à l'emploi d'un mot, d'une tournure ou d'une forme héritée du moyen haut allemand. Ainsi un afflux d'adverbes et conjonctions de subordination d'origine hébraïque et slave est venu compenser la perte ou le déclin des tournures correspondantes du moyen haut allemand. Dans certains cas, la sélection inhérente aux processus de koinéisation n'a pas joué et on voit coexister au sein du système des solutions accumulées au fil des siècles. Ainsi la proposition subordonnée concessive peut être introduite au moyen de la locution conjonctive ven oykh "quand bien même" où l'on reconnaît deux éléments hérités du moyen haut allemand: ven < wenne "quand" et oykh < ouch "aussi, même". Cette locution connaît une variante ven afile où l'élément germanique oykh a été remplacé par son exact équivalent hébreu afile < afilu. Dans un autre registre stylistique, on peut recourir à la conjonction hébraïque hagam où l'on reconnaît la locution conjonctive de l'hébreu michnaïque ha-gam she- "bien que" amputée de la conjonction de subordination she-. Ces solutions héritées du moyen haut allemand et/ ou de l'hébreu michnaïque sont souvent remplacées par khotsh où l'on

\footnotetext{
10 L'aoriste polonais a disparu dès le XIVe-XVe siècle (Vaillant, 1966: III, 62). À la même époque, l'imparfait polonais était également en pleine décadence (ibid.: III, 72). Par conséquent, le seul passé conservé en polonais est le parfait périphrastique (ibid.: III, 88).

11 En hébreu biblique, les valeurs du perfectif ne sont pas restreintes au passé. Mais en hébreu michnaïque, rabbinique et médiéval, ce perfectif peut être considéré comme un passé de plein droit.
} 
reconnaît la conjonction polonaise $c h o c$ [xots']. À vrai dire, ces différents moyens d'exprimer la concession ne sont pas complètement interchangeables. Le slavisme khotsh correspond au degré le plus fruste de la langue, cependant que hagam et ven afile relèvent d'un niveau de langage plus choisi. Cette stratification sociolinguistique entre les diverses composantes du yiddish révèle que la koinéisation survenue en Ashkenaz II à la fin du Moyen Age et au début des Temps modernes a dû faire intervenir des dynamiques qui intéressent directement la question de l'ethnogenèse des Juifs d'Europe de l'Est.

\section{LA KOINÉISATION SUR FOND D'ETHNOGENÈSE}

Pour reconstituer la dynamique des interférences hébraïques et slaves dans le processus de koinéisation du yiddish oriental, il importe de saisir les enjeux extralinguistiques des transformations internes à la langue. L'exemple des conjonctions ou locutions conjonctives à valeur concessive révèle qu'une stratification implicite sépare l'emploi des tournures hébraïques de la solution directement empruntée à l'adstrat polonais. La koinéisation du protoyiddish oriental aurait donc fait intervenir deux tendances antagonistes: d'une part, une très grande perméabilité à l'environnement linguistique slavophone; d'autre part, un raidissement de l'élite studieuse qui pouvait se targuer d'une origine juive allemande (et donc authentiquement ashkénaze). La crispation identitaire de cette aristocratie du savoir se serait donc manifestée non pas par une affirmation de la germanité de la koinè judéo-allemande cristallisée ou en voie de cristallisation, mais par un pédantisme de clerc consistant à recourir à une quantité extraordinaire d'hébraïsmes au sein même du vernaculaire yiddish.

Pour rendre compte de la concomitance des deux tendances apparemment contradictoires de la slavisation et de l'hébraïsation, on pourrait en outre invoquer un facteur qui a dû certainement jouer lors des premières décennies du contact linguistique entre Juifs émigrés d'Allemagne et Juifs slavophones autochtones. Comme il arrive souvent lorsque des Juifs d'horizon linguistique différent se rencontrent, la seule langue commune était le superstrat cultuel et culturel hébreu. Le choc de la rencontre entre ces deux rameaux du peuple juif aurait donc été en partie amorti par le recours à une língua franca qui n'était autre que l'hébreu. C'est ce qui expliquerait pourquoi de toutes les judéolangues, le yiddish oriental est de loin la plus riche en hébraïsme. La koinéisation qui est la base du protoyiddish oriental aurait non seulement abouti à une simplification de la base germanique et à une slavisation du système linguistique, mais aussi à l'intégration de la língua franca hébraïque en usage à la fin du Moyen Age entre les érudits ashkénazes et leurs homologues juifs slaves. Cet engloutissement de l'hébreu par la koinè yiddish orientale en voie de cris 
tallisation rappelle le processus en vertu duquel, à peu près à la même époque (XIV-XV ${ }^{\mathrm{e}}$ siècles) la koinè moyen-anglaise absorba ce qui restait de la poche récessive constituée par le dialecte anglo-normand.

Le vrai gagnant de cette concurrence entre des identités linguistiques hétérogènes est somme toute la koinè supradialectale allemande qui se cristallisa en terres slaves. Outre le rôle de língua franca que l'hébreu dut assumer au commencement du contact linguistique, la minimisation du décalage entre les communautés linguistiques hétéroglottes passa par l'ashkénazisation des Juifs slaves et par la slavisation des Ashkénazes. Cette convergence entre la germanité et la slavité se solda par l'adoption de la koinè protoyiddish orientale par les allophones d'expression slave compensée en partie par la slavisation des structures profondes de cette même koinè. Le schéma en vertu duquel une minorité prestigieuse d'immigrants absorbe culturellement une majorité silencieuse d'autochtones est bien attesté au cours de l'histoire du peuple juif. Lorsque les expulsés d'Espagne arrivèrent en Grèce et en Turquie, ils absorbèrent presque ${ }^{12}$ entièrement les Juifs romaniotes hellénophones pourtant supérieurs en nombres. Ceux des expulsés qui se rendirent au nord du Maroc et dans l'Oranais hispanisèrent en profondeur les communautés locales de Tanger, Tétouan, Ceuta, Melilla et Oran. De la même façon les immigrants de Rhénanie et de Franconie germanisèrent des masses juives de langue slave en imposant leur koinè judéoallemande, sociolecte d'un groupe prestigieux, et en remplaçant la prononciation judéo-byzantine de l'hébreu par la prononciation ashkénaze (Altbauer, 1992 : 22). De fait, la tradition intellectuelle et spirituelle du judaïsme allemand au Moyen Age a laissé beaucoup plus de trace dans l'histoire juive que le judaïsme d'expression slave, émanation lointaine du judaïsme byzantin. Ce synécisme des deux composantes du judaïsme d'Europe orientale - les immigrés et les autochtones - est la seule façon de rendre compte de l'extraordinaire boom démographique enregistré par le judaïsme ashkénaze au début des temps modernes. Alors qu'aux alentours de l'an Mil, les Juifs d'Europe non-méditerranéenne ne constituaient que $10 \%$ de la population juive mondiale, ils devinrent une majorité écrasante dès le XVII ${ }^{\mathrm{e}}$ siècle au moins. ${ }^{13}$ Même la saignée démographique provoquée par les terribles massacres de Bohdan Chmielnicki en 1648 ne remirent pas en question l'extraordinaire vitalité démographique du judaïsme ashkénaze.

$\mathrm{Au}$ terme de notre reconstruction des implications linguistiques de la cristallisation d'une identité juive d'Europe orientale résultant du synécisme entre Juifs allemands et Juifs slavophones, il apparaît clairement que pour une grande

\footnotetext{
12 Seules quelques poches d'hellénophonie subsistèrent au nord de la Grèce (Ioanina), dans l'Eubée ainsi que parmi les karaïtes stamboulis.

${ }^{13}$ En 1939, à la veille de la Shoah, 85 \% des Juifs étaient ashkénazes.
} 
partie de l'ethnos juif d'Europe orientale, le slave a longtemps constitué un substrat plutôt qu'un adstrat. En témoigne le fait que les noms des parents et des grands-parents en yiddish oriental sont presque tous d'origine slave: tate "père" a largement concurrencé la continuation du moyen haut allemand vater $;{ }^{14}$ mame "mère" a remplacé muoter; zeyde "grand-père" est évidemment un slavisme, même si la filière exacte de l'emprunt est difficile à reconstituer (Weinreich, 1980: 547-549); bobe "grand-mère" est également d'origine slave. Seul le nom des oncles et des tantes prolonge le legs germanique du yiddish: feter "oncle" continue le moyen haut allemand vetere dont le sens n'était pas encore "cousin" comme en allemand moderne; mume "tante" est l'aboutissement de mиоте. En revanche, les noms de filiation sont tout à fait germaniques: zon "fils"; tokhter "fille"; eynikel "petit-fils, petite-fille". Seul le nom du neveu et de la nièce est d'origine slave: plemenik/ plemenitse. Cette symétrie spéculaire qui veut que les noms de parenté soient tous d'origine slave sauf le nom de l'oncle et de la tante et que les noms de filiation soient tous d'origine germanique sauf le nom du neveu et de la nièce ne fait que corroborer le principe en vertu duquel les membres les plus directs de la famille sont appelés au moyen de termes empruntés au substrat slave, celui auquel les enfants issus de la fusion entre l'élément ashkénaze stricto sensu et la base judéo-slave étaient le plus constamment exposés au cours de leurs tout premiers échanges linguistiques.

Outre le critère de l'âge, on pourrait aussi faire intervenir celui du genre pour rendre compte des tendances contradictoires et complémentaires de l'hébraïsation et de la slavisation. Dans un monde où la prière synagogale et l'étude de la Torah étaient l'apanage exclusif des hommes, il était naturel que la composante hébraïque, marque de culture et d'élitisme social, fût identifiée avec le pôle masculin. En revanche, les femmes étaient plus proches du substrat/ adstrat slave qui servait notamment à désigner les réalités de la vie quotidienne. En outre, il semble qu'à partir du XVII ${ }^{\mathrm{e}}$ siècle, date à laquelle le monde juif d'Europe orientale connut un regain de religiosité et d'ardeur pour l'étude, beaucoup d'hommes déléguèrent aux femmes le soin de subvenir aux besoins de la famille. Cette répartition des tâches au sein du ménage explique en partie pourquoi les femmes juives d'Europe orientale maitrisaient les adstrats slaves (polonais, biélorusse, ukrainien) mieux que ne le faisaient leurs maris ou leurs fils, enfermés dans le monde protecteur de la maison d'étude.

La perméabilité du monde féminin à l'égard du substrat/adstrat slave peut être mise en relation avec l'extraordinaire profusion de diminutifs affectueux d'origine slave en yiddish oriental. À côté de $\{-1\} /\{-1$ e $\}$ qui est d'origine ger

\footnotetext{
14 La forme foter est certes attestée en yiddish oriental, mais elle relève d'un niveau de langue un peu pédant qui n'est assurément pas celui du langage enfantin.
} 
manique, le yiddish regorge de suffixes à valeur affective ou affectueuse qu'il a empruntés au slave: -ke, -inke, -uk, -ak, -atsh, -ulye, -enyu, -tshik, -nik (Weinreich, 1980: 531). Dans de nombreuses langues, l'abus de diminutifs caractérise le style mièvre connu sous le nom de baby talk, ce langage spécial que les femmes utilisent dans leurs échanges verbaux avec les enfants. Du point de vue synchronique, cette valeur stylistique particulière a été atténuée par l'inflation des diminutifs en yiddish oriental. Du point de vue diachronique, toutefois, la slavisation du protoyiddish oriental semble en grande partie liée à l' extension du langage féminin et enfantin à l'ensemble de la communauté linguistique.

\section{CONCIUSIONS}

Le processus de koinéisation du protoyiddish oriental en terres slaves n'est que l'expression linguistique de la fusion de deux identités: celle des émigrés juifs allemands et celle des Juifs slavophones. Bien que déracinés de leur contrée d'origine, les Ashkénazes venus de Rhénanie, de Franconie ou de Bavière parvinrent à imposer leur koiné à leurs coreligionnaires autochtones. Cette acculturation de l'identité autochtone à celle l'émigré eut du reste une contrepartie dans l'autre sens, puisque l'adstrat slave exerça une influence profonde sur la dynamique de la cristallisation d'une langue commune à tous les émigrés d'une part, aux Juifs locaux et aux Juifs émigrés d'une part. La profusion d'hébraïsmes qui caractérise cette langue nouvelle pourrait résulter d'une forme de snobisme intellectuel de la part d'érudits ashkénazes ressentant une complexe de supériorité vis-à-vis des Juifs slavisés et éprouvant comme une menace la pression de cette identité juive slavophone au sein de leur communauté, voire même de leur famille. On peut également penser que les premières phases du contact entre germanophones et slavophones rendirent nécessaire le recours à une língua franca consistant à utiliser les mots du superstrat cultuel et culturel hébreu.

Quoi qu'il en soit, le yiddish oriental apparaît comme une entité cohérente et bien cimentée malgré l'hétérogénéité de ses composantes et en dépit de sa dialectalisation secondaire en deux rameaux principaux: celui du nordest qui comprend la Lithuanie au sens large (Biélorussie incluse); celui du sud-est qui regroupe la Pologne et l' Ukraine. Il est d'ailleurs tentant d'attribuer les différences linguistiques et culturelles entre l'identité juive lithuanienne d'une part et l'identité juive polono-ukrainienne d'autre part à des disparités dans le rythme et l'intensité de la slavisation à l'époque de l'ethnogenèse et de la koinéisation. Cette question est riche d'enjeux qui dépassent la seule linguistique. De fait derrière l'opposition dialectale entre le rameau du nord-est et celui du sud-est se profile une différence identitaire 
fondamentale comme celle qui distingue les misnagdim lithuaniens opposés au hassidisme et les hasidim polono-ukrainiens. Même si cette distinction date seulement du début du XVIII ${ }^{\mathrm{e}}$ siècle, il est probable qu'elle plonge ses racines bien plus loin et qu'elle soit imputable à des disparités fondamentales dans l'intensité de la slavisation.

\section{BIBLIOGRAPHIE}

ALTBAUER, Moshé. The Five Biblical Scrolls in a Sixteenth-Century Jewish Translation into Bielorussian (Vilinius Codex 262), Jérusalem, The Israel Academy of Sciences and Humanities, 1992.

EGGERS Eckhard. Sprachwandel und Sprachmischung im Jiddischen, Francfort-sur-le Main, Peter Lang, 1998.

GUGGENHEIM-GRINBERG, Florence. "The Horse Dealers' Language of the Swiss Jews in Endingen and Lengnau". In: Uriel Weinreich (éd.), The Field of Yiddish: Studies in Language, Folklore, and Literature, New York, Linguistic Circle of New York, 1954, p. 48-62.

HERZOG, Marvin I. The Yiddish Language in Northern Poland: Its Geography and History, La Haye, Mouton, 1965.

JACOBS, Neil J. Economy in Yiddish Vocalism: A Study in the Interplay of Hebrew and Non-Hebrew Components, Wiesbaden, Otto Harrassowitz, 1990.

KING, Robert D. "Proto Yiddish Morphology". In: Language \& Communication, Vol. 7, Supplement, 1987, p. 73-81.

KURASZKIEWICZ, Wladyslaw. Zarys dialectologii wschodnioslowianskiej, $2^{\mathrm{e}}$ éd., Varsovie, Panstwowe Wydawnictwo Naukowe, 1963.

PRINCE, Ellen. "Yiddish as a Contact Language". In: Norval Smith et Tonjes Veestra (éd.), Creolization and Contact, Amsterdam-Philadelphie, John Benjamins, 2001, p. 263-289.

SCHUCHARDT, Hugo. Slawo-Deutsches und Slawo-Italienisches, Graz, Leuschner \& Lubensky, 1884 (repr. Munich, Wilhelm Fink, 1971).

SPITZER, Leo (éd.). Hugo Schuchardt-Brevier: Ein Vademecum der allgemeinen Sprachwissenschaft, 2e éd., Halle, Max Niemeyer, 1928.

SIEGEL, Jeff. "Koines and Koineization". In : Language in Society, 14, p. 357-378, 1985.

VAILLANT, André. Grammaire comparée des langues slaves, 5 vol., Paris, Klincksieck, 1966.

WEINREICH, Max. History of the Yiddish Language, trad. Shlomo Noble, Chicago-Londres, The University of Chicago Press, 1980.

WEXLER, Paul. "Slavic Influence in the Grammatical Function of Three Yiddish Verbal Prefixes". In: Linguistics, 7, p. 83-93, 1964.

. "Yiddish - the Fiftheenth Slavic Language. A Study of Partial Language Shift from Judeo-Sorbian

to German". International Journal of the Sociology of Language, 91, 1991, p. 9-150.

. The Ashkenazic Jews: A Slavic-Turkic People in Search of a Jewish Identity, Columbus,

Slavica, 1993.

Two-tiered Relexification in Yiddish: Jews, Sorbs, Khazars, and the Kiev-Polessian Dialect, Berlin, Mouton de Gruyter, 2002. 